\title{
Hypertensive Crisis
}

National Cancer Institute

\section{Source}

National Cancer Institute. Hypertensive Crisis. NCI Thesaurus. Code C3122.

A severe, acute increase in blood pressure that may result in stroke or myocardial ischemia. 